\title{
Chemical activity induces dynamical force with global structure in a reaction-diffusion-convection system
}

\author{
Hitoshi Mahara, ${ }^{1}$ Koichi Okada, ${ }^{2}$ Atsushi Nomura, ${ }^{3}$ Hidetoshi Miike, ${ }^{4}$ and Tatsunari Sakurai ${ }^{5, *}$ \\ ${ }^{1}$ Nanotechnology Research Institute, National Institute of Advanced Industrial Science and Technology (AIST), \\ Tsukuba 305-8565, Japan \\ ${ }^{2}$ Organization for University Education, Yamaguchi University, Yamaguchi 753-8511, Japan \\ ${ }^{3}$ Faculty of Education, Yamaguchi University, Yamaguchi 753-8513, Japan \\ ${ }^{4}$ Graduate School of Science and Engineering, Yamaguchi University, Ube 755-8611, Japan \\ ${ }^{5}$ Graduate School of Science, Chiba University, Chiba 263-8522, Japan
}

(Received 13 April 2009; published 29 July 2009)

\begin{abstract}
We found a rotating global structure induced by the dynamical force of local chemical activity in a thin solution layer of excitable Belousov-Zhabotinsky reaction coupled with diffusion. The surface flow and deformation associated with chemical spiral waves (wavelength about $1 \mathrm{~mm}$ ) represents a global unidirectional structure and a global tilt in the entire Petri dish (100 mm in diameter), respectively. For these observations, we scanned the condition of hierarchal pattern selection. From this result, the bromomalonic acid has an important role to induce the rotating global structure. An interaction between a reaction-diffusion process and a surfacetension-driven effect leads to such hierarchal pattern with different scales.
\end{abstract}

DOI: 10.1103/PhysRevE.80.015306

\section{INTRODUCTION}

Functional order associated with self-organized and hierarchical pattern formation is observed in biological and chemical systems [1-4]. Some of these systems are used to convert chemical energy into mechanical work. Typical examples are the contraction of cardiac muscles induced by calcium waves [5], the molecular motor induced by adenosine triphosphate (ATP) [6,7], and so on. Complicated but coherent pattern dynamics, which cannot be understood within chemical reactions coupled with molecular diffusion, have also been revealed [8-18]. An excellent example is the swelling or shrinking motions of media made of $\mathrm{N}$-isopropylacrylamide (NIPA) gel, which depend on the concentrations of the oxidized and reduced catalysts (i.e., chemical pattern) in Belousov-Zhabotinsky (BZ) reaction $[8,9]$. Another example is the convective flow initiated by a nonuniform spatial distribution of surface tension produced by chemical activity, such as the movement of a droplet of BZ solution [10] and an accelerating single chemical wave [11-13].

A hierarchical self-organized order of superimposed spiral waves with different scales and mechanistic origins also appears in a system that connects two complex phenomena involving the coupling of a reaction-diffusion pattern with convection [14-17]. Chemical waves initiate a macroscopic propagating convective flow pattern with surface deformation, called a flow wave. Its wavelength is 50 times larger than that of the chemical waves. The pattern dynamics link two different hierarchical levels of structure formation, i.e., the reaction-diffusion pattern and the convection with surface deformation induced by the reaction-diffusion pattern. Chemomechanical transduction in a reaction-diffusionconvection system offers wide flexibility for designing a hierarchical structure in nonlinear systems far from equilibrium.

\footnotetext{
*tatsu@physics.s.chiba-u.ac.jp
}

PACS number(s): 47.54.-r, 47.20.Dr, 47.70.Fw, 82.40.Ck

Numerical computation of surface-tension-driven convection depending on the distribution of $\mathrm{BZ}$ pattern has been performed by solving reaction-diffusion-advection equations coupled with the Navier-Stokes equation with no surface (interface) deformation [19-23]. These studies suggest that the scale of convective flow corresponds to the scale of chemical patterns. The complexity of flow waves at larger scales arises from the fact that it is nontrivial to describe them by a model with coupling only between chemical activities and surfacetension-driven convection.

In this Rapid Communication, we observed surface flow directions and surface deformation associated with chemical spiral waves in a thin solution layer of BZ reaction with a free surface. From these observations, we demonstrate synchronization of convective flow and the rotating flow wave with surface deformation. We demonstrate a structure caused by interaction between the reaction-diffusion process and a surface-tension-driven effect with rotating surface motion via chemomechanical transduction in an excitable system.

\section{EXPERIMENT}

We performed experiments under the condition that only the rotating flow wave appeared in the container. Experimental conditions were as follows: $8.08 \mathrm{ml}$ of prepared BZ solution was poured into a $100-\mathrm{mm}$-diameter Petri dish, and the thickness of the BZ solution layer was about $1 \mathrm{~mm}$. The BZ solution was composed of $48 \mathrm{mM} \mathrm{NaBr}, 340 \mathrm{mM} \mathrm{NaBrO}$, $95 \mathrm{~m} M$ malonic acid, $378 \mathrm{~m} M \mathrm{H}_{2} \mathrm{SO}_{4}$, and $3.5 \mathrm{~m} M$ ferroin as the catalyst and indicator. The Petri dish was covered with a glass plate to prevent evaporation after a pair of chemical spiral cores had been initiated at its center. An air gap of about $20 \mathrm{~mm}$ existed between the surface of the solution and the glass plate. During the experiments, room temperature was maintained at $25 \pm 1{ }^{\circ} \mathrm{C}$.

Two types of observations were performed. First, we observed the direction of convective flow on the BZ solution surface at local points. For these observations, we mixed 
polystyrene particles into the solution; the particles had no influence on any chemical reactions $[14,15]$. These particles were illuminated by $\mathrm{He}-\mathrm{Ne}$ laser light. Particle motion was detected using a charge coupled device (CCD) camera through a microscope. At the same time, we observed chemical activity and flow wave patterns using another CCD camera with a $490 \mathrm{~nm}$ interference filter above the Petri dish. The selected optical wavelength corresponds to the maximum difference between the absorption of oxidized and reduced forms of the catalyst. Further details about the experimental equipment were reported earlier [15]. Second, we observed surface deformations of the BZ solution using a Fizeau interferometer (FT-60LD, Mizojiri). The observation area was $60 \mathrm{~mm}$ in diameter at the center of the Petri dish. Optical flat-base glass $(\lambda / 10)$ was used as cover glass to avoid fringe pattern deformations caused by the cover glass. Fringe patterns detected using the CCD camera and a video recorder were analyzed by an inverse cosine function method [24].

\section{RESULTS}

At the beginning of our experiments, a pair of spiral cores was initiated at the center of the Petri dish. The chemical spiral cores rotated with a period of about $18 \mathrm{~s}$ and became a source of chemical wave train, which propagated from the center to the edge of the Petri dish with a minimum wavelength about $1 \mathrm{~mm}$. At around $15 \mathrm{~min}$ after the spiral cores were initiated, a rotating flow wave appeared spontaneously. In this time, the chemical wave pattern had already filled the Petri dish. Time sequences of the rotating flow wave (represented as brighter regions) are shown in Fig. 1. The tip of the rotating flow wave was located near the chemical spiral cores. This rotating flow wave had a period of about $30 \mathrm{~s}$ and continued until $30 \mathrm{~min}$ after the spiral cores were initiated. Here, the direction of the observed surface flows at three points was overlaid on the time sequence of rotating flow waves in Fig. 1. The surface flow was always running, i.e., the velocity of surface flow was always nonzero, and the directions of the surface flow rotated continuously. This rotation had the same period as the rotating flow wave and was synchronized with the rotating flow wave pattern. When the flow wave pattern (brighter areas in Fig. 1) passed the observation points, surface flows were directed almost inward [see Figs. 1(b-3) and 1(c-4)]. On the other hand, when the flow wave pattern was located at the opposite side of the observation points, the surface flows were directed outward [see Figs. 1(b-3) and 1(c-2)].

Figure 2 shows surface deformations of the rotating flow wave. These surface deformations are based on the fringe patterns captured by a Fizeau interferometer. The global surface tilt was certainly not due to tilting of the Petri dish with respect to the ground. The vertical interval between the highest and lowest parts is about $3 \mu \mathrm{m}$. The normal vector of the tilting surface rotated with a period of about $30 \mathrm{~s}$, the same at that of the flow wave.

\section{DISCUSSION}

The two types of observation were combined to determine the entire surface flow structure of the rotating flow wave. In
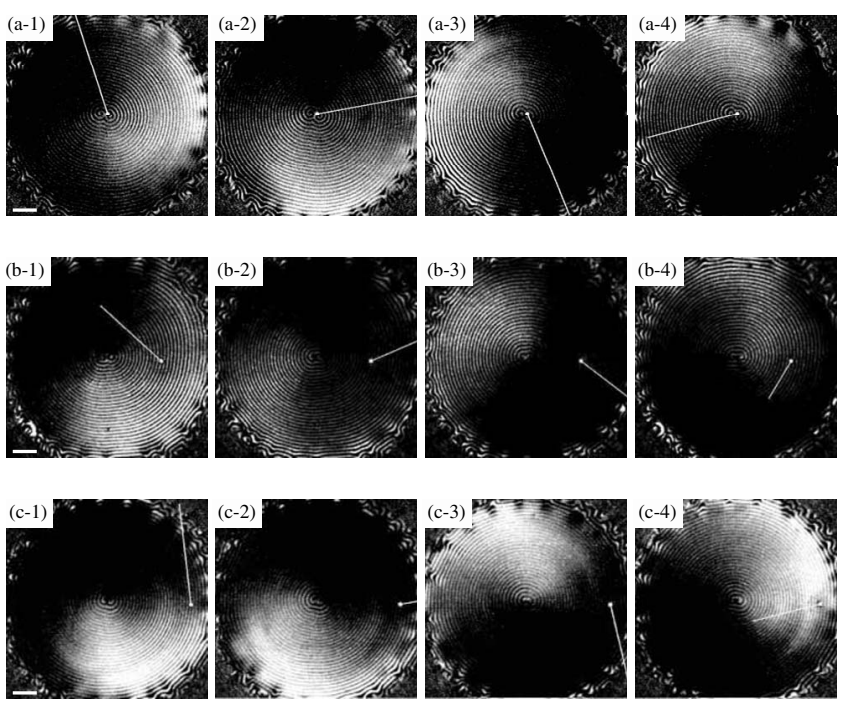

FIG. 1. Time series of flow wave patterns and flow direction on the solution surface. Pattern contrasts are emphasized so that only the flow wave pattern appears as a brighter area. The method of flow wave visualization is described in Ref. [14]. The white dots are the observation points of surface flow. The white lines indicate the direction of flow at the observation points; (a) $r=0 \mathrm{~mm}$, (b) $r$ $=33 \mathrm{~mm}$, and (c) $r=66 \mathrm{~mm} . r$ is the distance between the observation points and the chemical spiral cores. The direction of the flow wave rotation was random in each experiment. In this Rapid Communication, we used only clockwise rotating flow waves because the essential characteristics of both counterclockwise and clockwise rotating flow waves are the same. The time interval of the figures is about $8 \mathrm{~s}$. The scale bar in (a-1), (b-1), and (c-1) is 10 $\mathrm{mm}$. Movies of the rotating flow waves and surface flow are available [27].

the first experiments, surface flow directions were synchronized with the rotating flow wave pattern at each observation point, as shown in Fig. 1. The surface flow always pointed in the same relative direction against the rotating flow wave pattern. From this feature, it is natural to suppose that the surface flow structure was unique to the flow wave pattern. Thus, we should be able to determine the entire surface flow structure by superimposing the directions of surface flow over one period of the rotating flow wave onto the flow wave

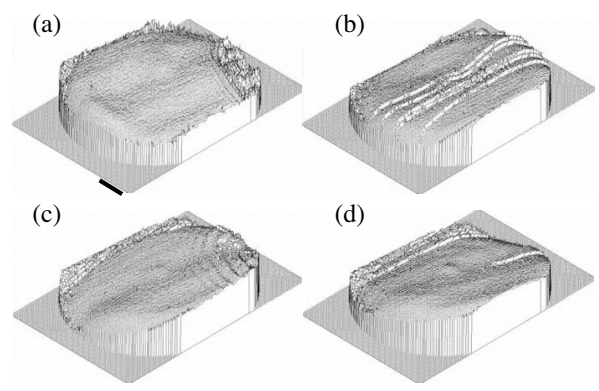

FIG. 2. Time series of analyzed surface deformation. The observation region is a circular region with a radius of $60 \mathrm{~mm}$. The time interval of the figures is about $7.5 \mathrm{~s}$. The distance between the highest and lowest parts is about $3 \mu \mathrm{m}$. The scale bar in (a) is 10 $\mathrm{mm}$. Movies of the fringe pattern and surface deformation are available [27]. 


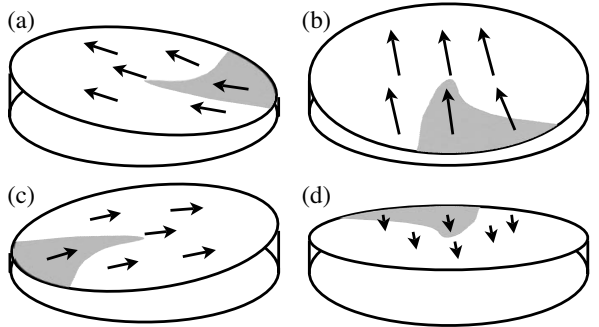

FIG. 3. Schematic view: time evolution of surface flow structure and surface deformation. The global structure of surface flows is determined from chemical wave pattern contrasts and surface flow direction in Fig. 1.

pattern in a single figure (Fig. 3). As seen in the determined surface flow structure, the surface flow was almost unidirectional globally, and the surface flow structure rotated periodically and was synchronized with the rotating flow wave.

From the second observation, global surface deformations were detected. We could not directly observe the relationship between the surface deformations and the flow wave pattern. However, the relationship between surface deformation and the surface flow structure should be inferred from the results of previous studies: the surface rises where surface flows converge [15]. In the first experiments, the surface flows ran globally from the brighter part to the darker part of the rotating flow wave pattern, so the surface flow converged to the darker part. Therefore, lower and higher areas of the surface should correspond to brighter and darker parts of the rotating flow wave pattern, respectively. From these two estimations, the surface structure induced by chemical spiral waves is finally seen to be working as a rotary surface deformation motion, as shown in Fig. 3.

In the rotary surface deformation motion, the global unidirectional surface flow and the globally tilted surface were rotating and synchronizing with the rotating flow wave patterns, which had a larger scale than that of the chemical wavelength. Thus, chemical species should have the same large-scale spatial gradient as the flow structure if they are to produce such a large-scale flow structure. Such chemicals should have longer time scale dynamics than the chemical oscillation period because if they have the same time scale as the chemical oscillations, they might produce a spatial structure at the same scale as the chemical wavelength. Many chemicals change slowly with repeating chemical oscillation in a BZ batch system. In particular, bromomalonic acid (BrMA), one such chemical species, is known as a surfactant [25]. Thus, there is some possibility that the spatial gradient of BrMA induces the flow wave patterns on the chemical pattern formation. To verify this possibility, we scanned the flow wave patterns in initial concentrations of BrMA and malonic acid (MA). The reason we chose an initial concentration of MA as an experimental parameter was that MA changes to BrMA through oscillations. In each experiment, the initial concentrations of the BZ solution were determined to the desired concentrations by using the five stock solutions and the chemical reaction formula described in Ref. [26]. Other conditions were the same as in the previous experiments. The spiral and circular flow wave patterns depended

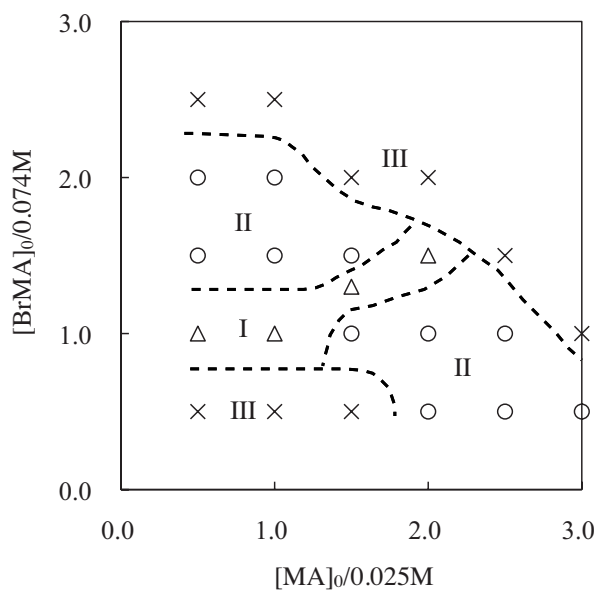

FIG. 4. Dependency of flow wave patterns on initial concentration of BrMA and MA. Regions I $(\triangle)$, II $(\bigcirc)$, and III $(X)$ show a rotating flow wave, a circular flow wave, and no flow wave, respectively. Here, the circular flow wave corresponds to the faint but bright shape of a circular structure with out- or in-going propagation. The detailed demonstration is in Refs. $[14,16]$. Each concentration was normalized by the experimental condition in Fig. 1.

on the initial concentrations of MA and BrMA, as shown in Fig. 4. For the appearance of these flow wave patterns, an upper limit of the BrMA initial concentration existed. Therefore, BrMA is an important component that makes the chemical-driven surface motion.

In summary, we observed surface flow directions and surface deformation of the rotating flow wave with two observation methods. From the results of these observations, we have determined the surface flow structure of the rotating flow wave. The structure was synchronized with the flow wave pattern and had a larger scale than the chemical wavelength.

The BrMA had an important role in inducing the flow wave pattern. The spatial gradients of the BrMA concentration change the spatial gradients of surface tension because the surface tension changes with BrMA concentration [25]. These surface tension deformations induce flow dynamics and flow wave patterns. Therefore, the pattern hierarchy induced by the $\mathrm{BZ}$ reaction, which has hierarchal time evolution, i.e., the time evolution hierarchy caused the hierarchal pattern: a flow wave pattern of $50 \mathrm{~mm}$ scale on the chemical patterns having a $1 \mathrm{~mm}$ wavelength. Chemomechanical transduction leads to a hierarchal order caused by interaction between the reaction-diffusion process and a surface-tensiondriven effect. Such chemomechanical transduction provides wide flexibility for designing hierarchal patterns with dynamical forcing.

\section{ACKNOWLEDGMENTS}

We thank to Dr. Tomohiko Yamaguchi and Dr. Osamu Inomoto for useful and invaluable discussions. This work was partly supported by Grants-in-Aid for Young Scientists A (Grant No. 18684022) from the Ministry of Education, Culture, Sports, Science and Technology of Japan. 
[1] J. P. Keener and J. Sneyd, Mathematical Physiology (Springer, New York, 1998).

[2] J. J. Tyson, K. A. Alexander, V. S. Manoranjan, and J. D. Murray, Physica D 34, 193 (1989).

[3] K. J. Lee, Nature (London) 369, 215 (1994).

[4] P. De Kepper, V. Castets, E. Dulos, and J. Boissonade, Physica D 49, 161 (1991).

[5] D. N. Bowser, T. Minamikawa, P. Nagley, and D. A. Williams, Biophys. J. 75, 2004 (1998).

[6] M. Schliwa and G. Woehlke, Nature (London) 422, 759 (2003).

[7] P.-D. Coureux, H. L. Sweeney, and A. Houdusse, EMBO J. 23, 4527 (2004).

[8] R. Yoshida, E. Kokufuta, and T. Yamaguchi, Chaos 9, 260 (1999).

[9] Y. Hara and R. Yoshida, J. Chem. Phys. 128, 224904 (2008).

[10] H. Kitahata, R. Aihara, N. Magome, and K. Yoshikawa, J. Chem. Phys. 116, 5666 (2002).

[11] J. A. Pojman, I. P. Nagy, and I. R. Epstein, J. Phys. Chem. 95, 1306 (1991).

[12] H. Miike, H. Yamamoto, S. Kai, and S. C. Müller, Phys. Rev. E 48, R1627 (1993).

[13] O. Inomoto, T. Ariyoshi, S. Inanaga, and S. Kai, J. Phys. Soc. Jpn. 64, 3602 (1995).

[14] K. Matthiessen and S. C. Muller, Phys. Rev. E 52, 492 (1995).

[15] T. Sakurai, E. Yokoyama, and H. Miike, Phys. Rev. E 56,
R2367 (1997).

[16] T. Sakurai, H. Miike, K. Okada, and S. C. Müller, J. Phys. Soc. Jpn. 72, 2177 (2003).

[17] T. Sakurai, O. Inomoto, H. Miike, K. Okada, and S. Kai, J. Phys. Soc. Jpn. 73, 485 (2004).

[18] M. S. Paoletti and T. H. Solomon, Phys. Rev. E 72, 046204 (2005).

[19] K. Matthiessen, H. Wilke, and S. C. Müller, Phys. Rev. E 53, 6056 (1996).

[20] Y. Wu, D. A. Vasquez, B. F. Edwards, and J. W. Wilder, Phys. Rev. E 51, 1119 (1995).

[21] M. Diewald, K. Matthiessen, S. C. Müller, and H. R. Brand, Phys. Rev. Lett. 77, 4466 (1996).

[22] H. Kitahata and K. Yoshikawa, Physica D 205, 283 (2005).

[23] L. Rongy and A. De Wit, J. Chem. Phys. 124, 164705 (2006).

[24] K. Okada, E. Yokoyama, and H. Miike, Electron. Commun. Jpn., Part 2: Electron. 90, 61 (2007).

[25] O. Inomoto, K. Abe, T. Amemiya, T. Yamaguchi, and S. Kai, Phys. Rev. E 61, 5326 (2000).

[26] H.-D. Försterling, L. Stuk, A. Barr, and W. D. McCormick, J. Phys. Chem. 97, 2623 (1993).

[27] See EPAPS Document No. E-PLEEE8-80-R07906 for movies of the rotating flow waves and surface flow. For more information on EPAPS, see http://www.aip.org/pubservs/ epaps.html. 